Volume 70, Number 1, Pages 555-568(2021)

DOI: $10.31801 /$ cfsuasmas.798620

ISSN 1303-5991 E-ISSN 2618-6470

Received by the editors: September 22, 2020; Accepted: February 10, 2021

\title{
AN APPROACH FOR DESIGNING A SURFACE PENCIL THROUGH A GIVEN GEODESIC CURVE
}

\author{
Gülnur ŞAFFAK ATALAY ${ }^{1}$, Fatma GÜLER ${ }^{2}$, Ergin BAYRAM ${ }^{2}$, and Emin KASAP 2 \\ ${ }^{1}$ Departmant of Mathematics Education,Ondokuz Mayis University, Samsun 55139, TURKEY \\ ${ }^{2}$ Departmant of Mathematics, Ondokuz Mayis University, Samsun 55139, TURKEY
}

\begin{abstract}
In the present paper, we propose a new method to construct a surface interpolating a given curve as the geodesic curve of it. Also, we analyze the conditions when the resulting surface is a ruled surface. In addition, developablity along the common geodesic of the members of surface family are discussed. Finally, we illustrate this method by presenting some examples.
\end{abstract}

\section{INTRODUCTION}

One of the frames defined for a space curve is the rotation minimizing frame, denoted by $\{T, U, V\}$. Here $\mathrm{T}$, the tangent of the curve; $\mathrm{U}$ and $\mathrm{V}$ are normal plane vectors that do not show instantaneous rotation around $T$. RMF's have a very important place in practice due to their minimum bending such that; curve constrained free form deformation [1-6], sweeping surface modeling [7-10], modeling of generalized cylinders and tree branches [11 - 14] are very interesting in computer graphics, including visualization. In addition, streamlines and tubes [15-17] play an important role in simulation of ropes and strings [18] and motion design and control [19]. One of the alternative frames that can be installed on the curve is the Bishop frame [20]. As it is known, the most known frame that can be installed on a curve in differential geometry is the Frenet frame (see [21] for details). However, since the Frenet frame can not be defined if the curvature is zero, the RMF frame is more useful than this frame [22].

One of the important curves on the surface is geodesic curves, and geodesic curves are closely related to Einstein's theory of relativity. In this sense, geodesics represent the paths of free falling objects. The concept of geodesic also finds its place

2020 Mathematics Subject Classification. 53A04, 53A05.

Keywords and phrases. Geodesic curve, parametric surface, rotation minimizing frame.

凶 gulnur.saffak@omu.edu.tr-Corresponding author; f.guler@omu.edu.tr;

ergin.bayram@omu.edu.tr; kasape@omu.edu.tr

(D) 0000-0003-4168-1642; 0000-0002-5107-8436; 0000-0003-2633-0991; 0000-0002-5017-5170.

(C)2021 Ankara University Communications Faculty of Sciences University of Ankara-Series A1 Mathematics and Statistics 
in various industrial applications, such as tent manufacturing, cutting and painting path, fiberglass tape windings in pipe manufacturing, textile manufacturing [23-28]. Deng, B., expressed the use of geodesic curves on the surface in architecture with various examples (for details, see [29] ). It is known by most people that the shortest distance between two points is a straight line. However, in differential geometry, this is said differently such that the shortest distance according to the Euclidean metric is expressed in geodesics. Geodesic curves are expressed as the extreme value of the distance function in some spaces. Geodesics are curves along which geodesic curvature vanishes. Contrary to traditional studies, the problem of finding a surface that passes through a given curve and accepts this curve as a special curve was first introduced by Wang et.al. [28] in Euclidean 3-space. A common geodesic surface family is defined by giving necessary and sufficient conditions for a given curve to be geodesic on a surface. Wang et al. stated that the data they obtained as a result of this study can be used in shoe and dress design in real life. Kasap et.al. [30] generalized the work of Wang by introducing new types of marching-scale functions, coefficients of the Frenet frame appearing in the parametric representation of surfaces. Also, surfaces with common geodesic in Minkowski 3-space have been the subject of many studies. In [31], Kasap and Akyıldız defined surfaces with a common geodesic in Minkowski 3-space and gave the sufficient conditions on marching-scale functions so that the given curve is a common geodesic on that surfaces. Şaffak and Kasap [32,33] studied family of surfaces with a common null geodesic and null asymptotic. Bayram et al. [34] studied parametric surfaces which possess a given curve as a common asymptotic. In [35], Bayram and Bilici studied surface family possessing an involute of a given curve as an asymptotic curve. In addition, they specifically stated their work for ruled surfaces and gave the conditions to be developable within them. Also, recently Atalay [36, 37] studied surface family possessing a Mannheim pair of a given curve as an asymptotic and geodesic curve. She, express necessary and sufficient conditions for that curve with above property and present natural results for such ruled surfaces. In addition, she specially provided the necessary and sufficient conditions to be developable through these ruled surfaces. In [38], Ayvacı finded a surface family possessing the Mannheim-B pair of given curve as an asymptotic and geodesic curve in Euclidean 3-space. She used Bishop frame of the given curve to solve this problem. Atalay and Ayvacı [39], derived the necessary and sufficient condition for Bertrand B-pair of a given curve to be geodesic curve on a surface in Euclidean 3-space. However, they solved the problem using Bishop-II frame of the given curve.

In this paper, we obtain the necessary and sufficient condition for a given curve to be both isoparametric and geodesic on a parametric surface depending on the RMF. Furthermore, we show that there exists ruled surfaces possessing a given curve as a common geodesic curve and present a criteria for these ruled surfaces to be developable ones. We only study curves with an arc length parameter because such 
a study is easy to follow; if necessary, one can obtain similar results for arbitrarily parameterised regular curves.

\section{Preliminaries}

A parametric curve $\beta(s), L_{1} \leq s \leq L_{2}$ is a curve on a surface $\Phi=\Phi(s, t)$ in $I R^{3}$ that has a constant $s$ or $t$-parameter value. In this paper, $\beta^{\prime}(s)$ denotes the derivative of $\beta$ with respect to arc length parameter $s$ and we assume that $\beta(s)$ is a regular curve, i.e. $\beta^{\prime}(s) \neq 0$. For every point of $\beta(s)$, if $\beta^{\prime \prime}(s) \neq 0$, the set $\{T(s), N(s), B(s)\}$ is called the Frenet frame along $\beta(s)$, where $T(s)=$ $\beta^{\prime}(s), N(s)=\beta^{\prime \prime}(s) /\left\|\beta^{\prime \prime}(s)\right\|$ and $B(s)=T(s) \times N(s)$ are the unit tangent, principal normal, and binormal vectors of the curve at the point $\beta(s)$, respectively. Derivative formulas of the Frenet frame is governed by the relations

$$
\left\{\begin{array}{c}
T^{\prime}(s)=\kappa(s) N(s) \\
N^{\prime}(s)=-\kappa(s) T(s)+\tau(s) B(s) \\
B^{\prime}(s)=-\tau(s) N(s)
\end{array}\right.
$$

where $\kappa(s)=\left\|\beta^{\prime \prime}(s)\right\|$ and $\tau(s)=\frac{\operatorname{det}\left(\beta^{\prime}(s), \beta^{\prime \prime}(s), \beta^{\prime \prime \prime}(s)\right)}{\left\|\beta^{\prime}(s) \times \beta^{\prime \prime}(s)\right\|}$ are called the curvature and torsion of the curve $\beta(s)$, respectively [40].

Another useful frame along a curve is rotation minimizing frame. They are useful in animation, motion planning, swept surface constructions and related applications where the Frenet frame may prove unsuitable or undefined. A frame $\{T(s), U(s), V(s)\}$ among the frames on the curve $\beta(s)$ is called rotation minimizing if it is the frame of minimum twist around the tangent vector $T$. $\{T(s), U(s), V(s)\}$ is an RMF if

$$
\left\{\begin{array}{l}
U^{\prime}(s)=-\left(U(s) \cdot r^{\prime \prime}(s)\right) r^{\prime}(s) \\
V^{\prime}(s)=-\left(V(s) \cdot r^{\prime \prime}(s)\right) r^{\prime}(s)
\end{array}\right.
$$

where "." denotes the standard inner product in $I R^{3}$ [41]. Observe that such a pair $U$ and $V$ is not unique; there exist a one parameter family of RMF's corresponding to different sets of initial values of $U$ and $V$. According to Bishop [20], a frame is an RMF if and only if each of $U^{\prime}(s)$ and $V^{\prime}(s)$ is parallel to $T(s)$. Equivalently,

$$
\left\{\begin{array}{c}
U^{\prime}(s) \cdot V(s) \equiv 0 \\
V^{\prime}(s) \cdot U(s) \equiv 0
\end{array}\right.
$$

is the necessary and sufficient condition for the frame to be rotation minimizing [42].

There is a relation between the Frenet frame (if the Frenet frame is defined) and RMF, that is, $U$ and $V$ are the rotation of $N$ and $B$ of the curve $\beta(s)$ in the normal plane. Then,

$$
\left(\begin{array}{l}
U \\
V
\end{array}\right)=\left(\begin{array}{cc}
\cos \theta & -\sin \theta \\
\sin \theta & \cos \theta
\end{array}\right)\left(\begin{array}{c}
N \\
B
\end{array}\right)
$$




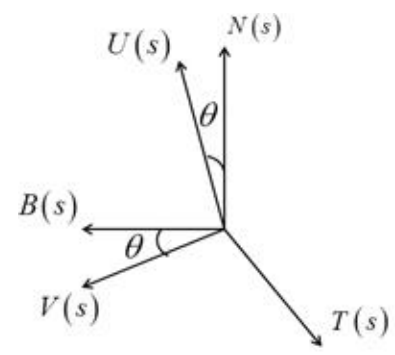

Figure 1. The Frenet frame $\{T(s), N(s), B(s)\}$ and the vectors $U(s), V(s)$.

where $\theta=\theta(s)$ is the angle between the vectors $N$ and $U$ (see Fig. 1), [43].

Equation (3) implies that $\{T(s), U(s), V(s)\}$ is an RMF if it satisfies the following relations

$$
U^{\prime}=-\kappa \cos \theta T, V^{\prime}(s)=\kappa \sin \theta T, \theta^{\prime}=-\tau .
$$

Note that $\{T(s), U(s), V(s)\}$ is defined along the curve $\beta(s)$ even if the curvature vanishes where the Frenet frame is undefined.

\section{Surfaces pencil With a Geodesic Curve}

Suppose we are given a 3-dimensional parametric curve $\beta(s), K_{1} \leq s \leq K_{2}$, in which $s$ is the arc length (regular and $\left\|\beta^{\prime}(s)\right\|=1, K_{1} \leq s \leq K_{2}$ ).

Surface pencil that interpolates $\beta(s)$ as a common curve is given in the parametric form as

$$
\Phi(s, t)=\beta(s)+p(s, t) T(s)+q(s, t) U(s)+r(s, t) V(s), K_{1} \leq s \leq K_{2}, L_{1} \leq t \leq L_{2},
$$

where $p(s, t), q(s, t)$ and $r(s, t)$ are $C^{1}$ functions. The values of the marching-scale functions $p(s, t), q(s, t)$ and $r(s, t)$ indicate, respectively; the extension-like, flexionlike and retortion-like effects, by the point unit through the time $t$, starting from $\beta(s)$.

Remark 1. Observe that choosing different marching-scale functions yields different surfaces possessing $\beta(s)$ as a common curve.

Our goal is to find the necessary and sufficient conditions for which the curve $\beta(s)$ is isoparametric and geodesic on the surface $\Phi(s, t)$. Firstly, as $\beta(s)$ is an isoparametric curve on the surface $\Phi(s, t)$, there exists a parameter $t_{0} \epsilon\left[L_{1}, L_{2}\right]$ such that

$$
p\left(s, t_{0}\right)=q\left(s, t_{0}\right)=r\left(s, t_{0}\right) \equiv 0, K_{1} \leq s \leq K_{2}, L_{1} \leq t_{0} \leq L_{2} .
$$

Secondly,the curve $\beta(s)$ is a geodesic curve on the surface $\Phi(s, t)$ there exist a parameter $t_{0} \epsilon\left[L_{1}, L_{2}\right]$ such that 


$$
n\left(s, t_{0}\right) \| N(s)
$$

where $n\left(s, t_{0}\right)$ is a normal along the curve $\beta(s)$ and $N(s)$ is a normal vector of $\beta(s)$.

The normal vector of $\Phi=\Phi(s, t)$ can be written as

$$
n(s, t)=\frac{\partial \Phi(s, t)}{\partial s} \times \frac{\partial \Phi(s, t)}{\partial t} .
$$

From equations (1) and (3), the normal vector can be expressed as

$$
\begin{aligned}
n(s, t)= & {\left[\begin{array}{c}
\frac{\partial r(s, t)}{\partial t}\left(p(s, t) \kappa(s) \cos \theta(s)+\frac{\partial q(s, t)}{d s}\right) \\
-\frac{\partial q(s, t)}{\partial t}\left(-p(s, t) \kappa(s) \sin \theta(s)+\frac{\partial r(s, t)}{d s}\right)
\end{array}\right] T(s) } \\
& +\left[\begin{array}{c}
\frac{\partial p(s, t)}{\partial t}\left(-p(s, t) \kappa(s) \sin \theta(s)+\frac{\partial r(s, t)}{d s}\right) \\
-\frac{\partial r(s, t)}{\partial t}\left(1-q(s, t) \kappa(s) \sin \theta(s)+r(s, t) \kappa(s) \sin \theta(s)+\frac{\partial p(s, t)}{d s}\right)
\end{array}\right] U(s) \\
& +\left[\begin{array}{c}
\frac{\partial q(s, t)}{\partial t}\left(1-q(s, t) \kappa(s) \cos \theta(s)+r(s, t) \kappa(s) \sin \theta(s)+\frac{\partial p(s, t)}{d s}\right) \\
-\frac{\partial p(s, t)}{\partial t}\left(p(s, t) \kappa(s) \cos \theta(s)+\frac{\partial q(s, t)}{d s}\right)
\end{array}\right] V(s) .
\end{aligned}
$$

Thus,

$$
n\left(s, t_{0}\right)=\Psi_{1}\left(s, t_{0}\right) T(s)+\Psi_{2}\left(s, t_{0}\right) U(s)+\Psi_{3}\left(s, t_{0}\right) V(s)
$$

where

$$
\begin{aligned}
& \Psi_{1}\left(s, t_{0}\right)=\frac{\partial r\left(s, t_{0}\right)}{\partial t}\left(p\left(s, t_{0}\right) \kappa(s) \cos \theta(s)+\frac{\partial q\left(s, t_{0}\right)}{d s}\right) \\
& -\frac{\partial q\left(s, t_{0}\right)}{\partial t}\left(-p\left(s, t_{0}\right) \kappa(s) \sin \theta(s)+\frac{\partial r\left(s, t_{0}\right)}{d s}\right), \\
& \Psi_{2}\left(s, t_{0}\right)=\frac{\partial p\left(s, t_{0}\right)}{\partial t}\left(-p\left(s, t_{0}\right) \kappa(s) \sin \theta(s)+\frac{\partial r\left(s, t_{0}\right)}{d s}\right) \\
& -\frac{\partial r\left(s, t_{0}\right)}{\partial t}\left(1-q\left(s, t_{0}\right) \kappa(s) \sin \theta(s)+r\left(s, t_{0}\right) \kappa(s) \sin \theta(s)+\frac{\partial p\left(s, t_{0}\right)}{d s}\right), \\
& \Psi_{3}\left(s, t_{0}\right)=\frac{\partial q\left(s, t_{0}\right)}{\partial t}\left(1-q\left(s, t_{0}\right) \kappa(s) \cos \theta(s)+r\left(s, t_{0}\right) \kappa(s) \sin \theta(s)+\frac{\partial p\left(s, t_{0}\right)}{d s}\right) \\
& -\frac{\partial p\left(s, t_{0}\right)}{\partial t}\left(p\left(s, t_{0}\right) \kappa(s) \cos \theta(s)+\frac{\partial q\left(s, t_{0}\right)}{d s}\right) .
\end{aligned}
$$

Remark 2. Because of equation (6) and by the definition of partial differentiation we have

$$
\frac{\partial p}{\partial s}\left(s, t_{0}\right)=\frac{\partial q}{\partial s}\left(s, t_{0}\right)=\frac{\partial r}{\partial s}\left(s, t_{0}\right) \equiv 0, t_{0} \epsilon\left[L_{1}, L_{2}\right], K_{1} \leq s \leq K_{2}
$$

According to remark above, we should have 


$$
\left\{\begin{array}{l}
\Psi_{1}\left(s, t_{0}\right) \equiv 0 \\
\Psi_{2}\left(s, t_{0}\right)=-\frac{\partial r}{\partial s}\left(s, t_{0}\right) \\
\Psi_{3}\left(s, t_{0}\right)=\frac{\partial q}{\partial s}\left(s, t_{0}\right)
\end{array}\right.
$$

Using equations (3), (8) and (9) we obtain

$$
\begin{aligned}
n\left(s, t_{0}\right)= & {\left[-\frac{\partial r}{\partial t}\left(s, t_{0}\right) \cos \theta(s)-\frac{\partial q}{\partial t}\left(s, t_{0}\right) \sin \theta(s)\right] N(s) } \\
& +\left[\frac{\partial q}{\partial t}\left(s, t_{0}\right) \cos \theta(s)-\frac{\partial r}{\partial t}\left(s, t_{0}\right) \sin \theta(s)\right] B(s) .
\end{aligned}
$$

Thus by (7) $\beta(s)$ is a geodesic on the surface $\Phi(s, t)$ if and only if

$$
\left\{\begin{array}{l}
\frac{\partial q}{\partial t}\left(s, t_{0}\right) \sin \theta(s)+\frac{\partial r}{\partial t}\left(s, t_{0}\right) \cos \theta(s) \neq 0 \\
\frac{\partial q}{\partial t}\left(s, t_{0}\right) \cos \theta(s)-\frac{\partial r}{\partial t}\left(s, t_{0}\right) \sin \theta(s)=0
\end{array}\right.
$$

Hence, we have following theorem:

Theorem 3. The necessary and sufficient condition for the curve $\beta(s)$ to be both isoparametric and geodesic on the surface $\Phi(s, t)$ is

$$
\left\{\begin{array}{l}
p\left(s, t_{0}\right)=q\left(s, t_{0}\right)=r\left(s, t_{0}\right) \equiv 0 \\
\frac{\partial q}{\partial t}\left(s, t_{0}\right)=\lambda(s) \sin \theta(s), \frac{\partial r}{\partial t}\left(s, t_{0}\right)=\lambda(s) \cos \theta(s), \lambda(s) \neq 0 \\
\theta^{\prime}(s)=-\tau(s)
\end{array}\right.
$$

Corollary 4. The sufficient condition for the curve $\beta(s)$ to be both isoparametric and geodesic on the surface $\Phi(s, t)$ is

$$
\left\{\begin{array}{l}
p\left(s, t_{0}\right)=q\left(s, t_{0}\right)=r\left(s, t_{0}\right) \equiv 0 \\
q(s, t)=\left(t-t_{0}\right) \lambda(s) \sin \theta(s), r(s, t)=\left(t-t_{0}\right) \lambda(s) \cos \theta(s), \lambda(s) \neq 0 \\
\theta^{\prime}(s)=-\tau(s)
\end{array}\right.
$$

\section{Ruled SuRface Pencil With a COMmon GeodesiC}

Theorem 5. Given an arc-length curve $\beta(s)$, there exists a ruled surface pencil possessing $\beta(s)$ as a common geodesic.

Proof. Choosing marching-scale functions as

$$
p(s, t)=\left(t-t_{0}\right) g(s), q(s, t)=\left(t-t_{0}\right) \lambda(s) \sin \theta(s), r(s, t)=\left(t-t_{0}\right) \lambda(s) \cos \theta(s),
$$

$\lambda(s) \neq 0$ and $\theta^{\prime}(s)=-\tau(s)$ equation (5) takes the following form of a ruled surface

$$
\Phi(s, t)=\beta(s)+\left(t-t_{0}\right)[g(s) T(s)+\lambda(s) \sin \theta(s) U(s)+\lambda(s) \cos \theta(s) V(s)],
$$

which satisfies equation (12) interpolating $\beta(s)$ as a common geodesic curve. 
Remark 6. Observe that, changing $g(s)$ and $\lambda(s)$ in equation (14) yields different ruled surfaces interpolating $\beta(s)$ as a common geodesic.

Corollary 7. Ruled surface (14) is developable if and only if $\tau(s)=g(s) \kappa(s)$ for some real valued function $g(s)$.

Proof. $\Phi(s, t)=\beta(s)+\left(t-t_{0}\right)[g(s) T(s)+\sin \theta(s) U(s)+\cos \theta(s) V(s)]$ is developable if and only if $\operatorname{det}\left(\beta^{\prime}, R, R^{\prime}\right)=0$, where $R(s)=g(s) T(s)+\sin \theta(s) U(s)+$ $\cos \theta(s) V(s)$.

Equations (1), (3) and (4) give

$$
\begin{aligned}
R^{\prime} & =g^{\prime} T+g T^{\prime}+\theta^{\prime} \cos \theta U+\sin \theta U^{\prime}-\theta^{\prime} \sin \theta V+\cos \theta V^{\prime} \\
& =g^{\prime} T+(g \kappa \cos \theta-\tau \cos \theta) U+(-g \kappa \sin \theta+\tau \sin \theta) V .
\end{aligned}
$$

Employing equation (15) in the determinant we get $\tau=g \kappa$, which completes the proof.

\section{Examples of generating surfaces with a COMmon geodesic Curve}

Example 8. Let $\beta(s)=\left(\frac{3}{5} \sin s, \frac{3}{5} \cos s, \frac{4}{5} s\right)$ be a unit speed curve. Then it is easy to show that

$$
T(s)=\left(\frac{3}{5} \cos s,-\frac{3}{5} \sin s, \frac{4}{5}\right), \kappa(s)=\frac{3}{5}, \tau(s)=-\frac{4}{5} .
$$

If we choose $\theta=\frac{4}{5} s$ we have

$$
\begin{gathered}
U^{\prime}=\left(-\frac{9}{25} \cos s \cos \left(\frac{4 s}{5}\right), \frac{9}{25} \sin s \cos \left(\frac{4 s}{5}\right),-\frac{12}{25} \cos \left(\frac{4 s}{5}\right)\right), \\
V^{\prime}=\left(\frac{9}{25} \cos s \sin \left(\frac{4 s}{5}\right),-\frac{9}{25} \sin s \sin \left(\frac{4 s}{5}\right), \frac{12}{25} \sin \left(\frac{4 s}{5}\right)\right)
\end{gathered}
$$

satisfying equation (4). By integration, we obtain

$$
\begin{aligned}
& U=\left(-\frac{1}{10} \sin \left(\frac{9 s}{5}\right)-\frac{9}{10} \sin \left(\frac{s}{5}\right),-\frac{1}{10} \cos \left(\frac{9 s}{5}\right)-\frac{9}{10} \cos \left(\frac{s}{5}\right),-\frac{3}{5} \sin \left(\frac{4 s}{5}\right)\right), \\
& V=\left(-\frac{1}{10} \cos \left(\frac{9 s}{5}\right)+\frac{9}{10} \cos \left(\frac{s}{5}\right),-\frac{9}{10} \sin \left(\frac{s}{5}\right)+\frac{1}{10} \sin \left(\frac{9 s}{5}\right),-\frac{3}{5} \cos \left(\frac{4 s}{5}\right)\right) .
\end{aligned}
$$

Now, $\{T(s), U(s), V(s)\}$ is an RMF since it satisfies equation (2). If we take $p(s, t) \equiv 0, q(s, t)=\sin \left(\frac{4 s}{5}\right)(\sin t-1), r(s, t)=\cos \left(\frac{4 s}{5}\right) \cos t, \lambda(s) \equiv-1$ and $t_{0}=\frac{\pi}{2}$,then equation (11) is satisfied. Thus, we obtain a member of the surface pencil with a common geodesic $\beta(s)$ 


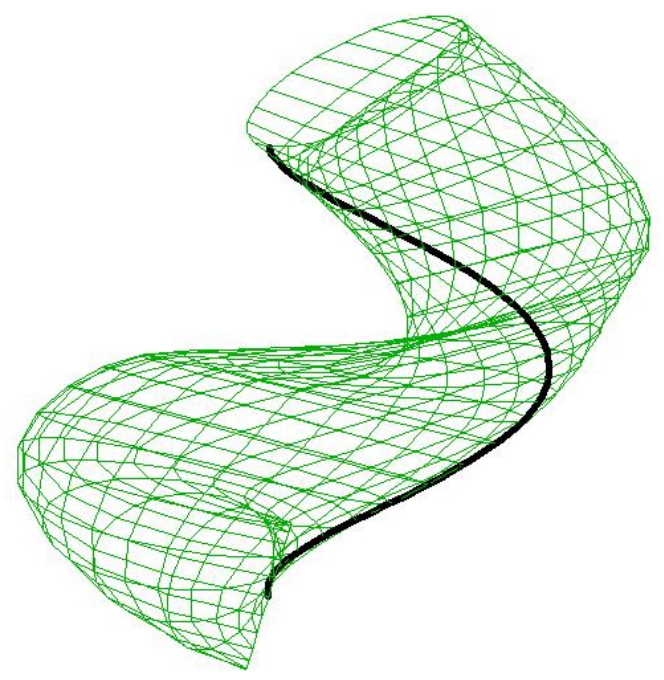

Figure 2. $\Phi_{1}(s, t)$ as a member of the surface pencil and its geodesic.

$$
\Phi_{1}(s, t)=\left(\begin{array}{c}
\frac{3}{5} \sin (s)-\sin \left(\frac{4 s}{5}\right)(\sin t-1)\left(-\frac{1}{10} \sin \left(\frac{9 s}{5}\right)-\frac{9}{10} \sin \left(\frac{s}{5}\right)\right) \\
+\cos \left(\frac{4 s}{5}\right) \cos t\left(-\frac{1}{10} \cos \left(\frac{9 s}{5}\right)-\frac{9}{10} \cos \left(\frac{s}{5}\right)\right) \\
\frac{3}{5} \cos (s)-\sin \left(\frac{4 s}{5}\right)(\sin t-1)\left(-\frac{1}{10} \cos \left(\frac{9 s}{5}\right)-\frac{9}{10} \cos \left(\frac{s}{5}\right)\right) \\
+\cos \left(\frac{4 s}{5}\right) \cos t\left(-\frac{9}{10} \sin \left(\frac{s}{5}\right)+\frac{1}{10} \sin \left(\frac{9 s}{5}\right)\right) \\
\frac{4 s}{5}-\frac{3}{5}\left(\sin ^{2}\left(\frac{4 s}{5}\right)(\sin t-1)+\cos ^{2}\left(\frac{4 s}{5}\right) \cos t\right)
\end{array}\right)
$$

where $0 \leq s \leq 2 \pi, 0 \leq t \leq 2 \pi$ (Fig. 2).

In equation. (14), if we take $g(s)=\frac{\tau(s)}{\kappa(s)}=-\frac{4}{3}$, then by Corollary 7 , we obtain the following developable ruled surface with a common geodesic $\beta(s)$ as

$$
\Phi_{2}(s, t)=\left(\begin{array}{c}
\frac{3}{5} \sin s+t\left(-\frac{4}{5} \cos s+\sin \left(\frac{4 s}{5}\right)\left(-\frac{1}{10} \sin \left(\frac{9 s}{5}\right)-\frac{9}{10} \sin \left(\frac{s}{5}\right)\right)\right) \\
+\cos \left(\frac{4 s}{5}\right)\left(-\frac{1}{10} \cos \left(\frac{9 s}{5}\right)+\frac{9}{10} \cos \left(\frac{s}{5}\right)\right), \\
\frac{3}{5} \cos s+t\left(\frac{4}{5} \sin s+\sin \left(\frac{4 s}{5}\right)\left(-\frac{1}{10} \cos \left(\frac{9 s}{5}\right)-\frac{9}{10} \cos \left(\frac{s}{5}\right)\right)\right) \\
+\cos \left(\frac{4 s}{5}\right)\left(-\frac{9}{10} \sin \left(\frac{s}{5}\right)+\frac{1}{10} \sin \left(\frac{9 s}{5}\right)\right), \\
\frac{4 s}{5}-\frac{3}{5} t
\end{array}\right)
$$

where $-2 \pi \leq s \leq 2 \pi, 0 \leq v \leq 2 \pi$ (Fig. 3 ).

Example 9. Let $\beta(s)=(\cos s, \sin s, 0)$ be a unit speed curve. It is obvious that $T(s)=(-\sin s, \cos s, 0), \quad \kappa(s)=1, \tau(s)=0$.

If we take $U(s)=(-\cos s,-\sin s, 0), V(s)=(0,0,1)$, then equation (2) is satisfied and $\{T(s), U(s), V(s)\}$ is an RMF. By choosing marching-scale functions as 


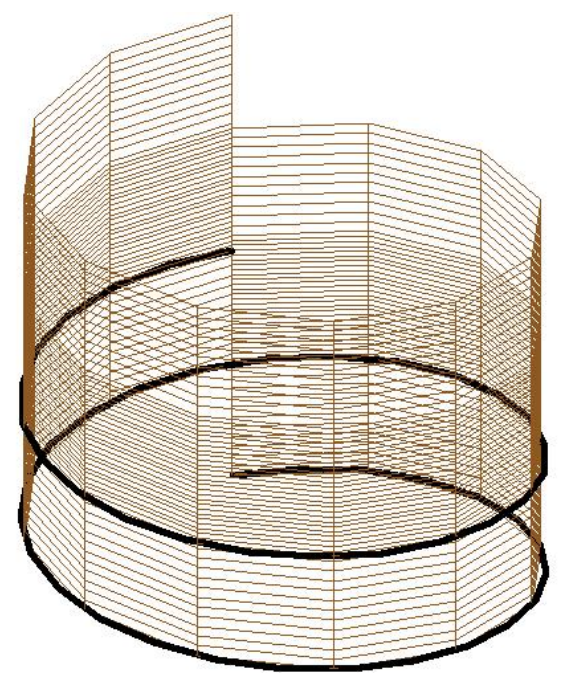

FiguRE $3 . \Phi_{2}(s, t)$ as a member of the developable ruled surface pencil and its geodesic.

$p(s, t) \equiv 0, q(s, t)=1-\cos t, r(s, t)=\sin t, \lambda(s) \equiv 1$ and $t_{0}=\theta=0$, then equation (11) is satisfied. Thus, we immediately obtain a member of the surface pencil with a common geodesic $\beta(s)$ as

$$
\Phi_{3}(s, t)=(\cos s \cos t, \sin s \cos t, \sin t),
$$

where $0 \leq s \leq 2 \pi, 0 \leq t \leq 2 \pi$ (Fig. 4).

For the same curve let us find a ruled surface. In equation (14), if we take $g(s)=\frac{\tau(s)}{\kappa(s)}=0$, then we obtain the following developable ruled surface with a common geodesic $\beta(s)$ as

$$
\Phi_{4}(s, t)=(\cos s, \sin s, t)
$$

where $-\pi \leq s \leq \pi,-1 \leq t \leq 1$ (Fig. 5 ).

If we take $g(s)=0$ and $t_{0}=\theta=\frac{\pi}{3}$ then, we obtain the following developable ruled surface with a common geodesic $\beta(s)$ as

$$
\Phi_{5}(s, t)=\left(\left(1-\frac{\sqrt{3}}{2}\left(t-\frac{\pi}{3}\right)\right) \cos s,\left(1-\frac{\sqrt{3}}{2}\left(t-\frac{\pi}{3}\right)\right) \sin s, \frac{1}{2}\left(t-\frac{\pi}{3}\right)\right),
$$

where $0 \leq s \leq 2 \pi, 0 \leq t \leq 2$ (Fig. 6 ). 


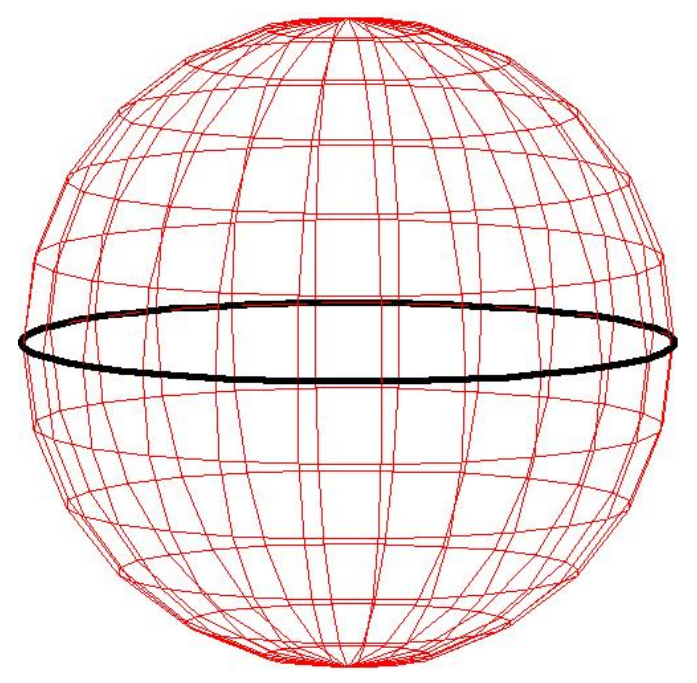

Figure $4 . \Phi_{3}(s, t)$ as a member of surface pencil and its geodesic.

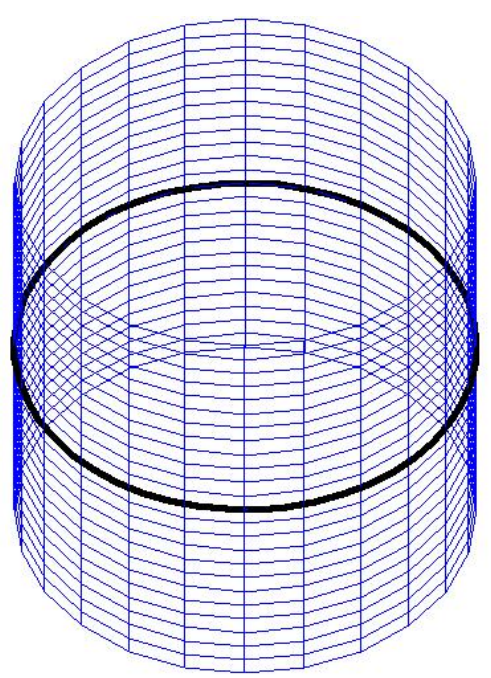

Figure 5. $\Phi_{4}(s, t)$ as a member of the developable ruled surface pencil and its geodesic curve. 


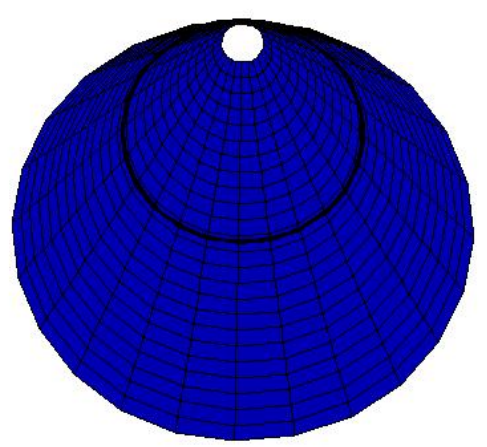

Figure $6 . \Phi_{5}(s, t)$ as a member of the developable ruled surface pencil and its geodesic curve.

Example 10. Let $\beta(s)=\left(\frac{\sqrt{3}}{2} \sin s, \frac{s}{2}, \frac{\sqrt{3}}{2} \cos s\right)$ be a unit speed curve. Then,

$$
T(s)=\left(\frac{\sqrt{3}}{2} \cos s, \frac{1}{2}, \frac{\sqrt{3}}{2} \sin s\right), \kappa(s)=\frac{\sqrt{3}}{2}, \tau(s)=-\frac{1}{2} .
$$

Using equations (2) and (4) we obtain $\theta=-\frac{s}{2}$ and

$$
\begin{gathered}
U=\left(-\frac{1}{4} \sin \left(\frac{3 s}{2}\right)-\frac{3}{4} \sin \left(\frac{s}{2}\right),-\frac{\sqrt{3}}{2} \sin \left(\frac{s}{2}\right),-\frac{1}{4} \cos \left(\frac{3 s}{2}\right)-\frac{3}{4} \cos \left(\frac{s}{2}\right)\right), \\
V=\left(\frac{1}{4} \cos \left(\frac{3 s}{2}\right)-\frac{3}{4} \cos \left(\frac{s}{2}\right), \frac{\sqrt{3}}{2} \cos \left(\frac{s}{2}\right), \frac{3}{4} \sin \left(\frac{s}{2}\right)-\frac{1}{4} \sin \left(\frac{3 s}{2}\right)\right) .
\end{gathered}
$$

Now, $\{T(s), U(s), V(s)\}$ is an RMF. If we take $p(s, t) \equiv 0, q(s, t)=s^{2} t \sin \left(\frac{s}{2}\right), r(s, t)=$ $s^{2} t \cos \left(\frac{s}{2}\right)$ and $t_{0}=0$, then equation (12) we have a member of the surface pencil with a common geodesic $\beta(s)$ as

$$
\Phi_{6}(s, t)=\left(\begin{array}{c}
\frac{\sqrt{3}}{2} \sin (s)+s^{2} t\left(\sin \left(\frac{s}{2}\right)\left(-\frac{1}{4} \sin \left(\frac{3 s}{2}\right)-\frac{3}{4} \sin \left(\frac{s}{2}\right)\right)\right. \\
\left.+\cos \left(\frac{s}{2}\right)\left(\frac{1}{4} \cos \left(\frac{3 s}{2}\right)-\frac{3}{4} \cos \left(\frac{s}{2}\right)\right)\right) \\
s+s^{2} t\left(\frac{\sqrt{3}}{2} \cos ^{2}\left(\frac{s}{2}\right)-\frac{\sqrt{3}}{2} \sin ^{2}\left(\frac{s}{2}\right)\right) \\
\frac{\sqrt{3}}{2} \cos (s)+s^{2} t\left(\sin \left(\frac{s}{2}\right)\left(-\frac{1}{4} \cos \left(\frac{3 s}{2}\right)-\frac{3}{4} \cos \left(\frac{s}{2}\right)\right)\right. \\
\left.+\cos \left(\frac{s}{2}\right)\left(\frac{3}{4} \sin \left(\frac{s}{2}\right)-\frac{1}{4} \sin \left(\frac{3 s}{2}\right)\right)\right)
\end{array}\right)
$$

where $0<s \leq 2,-1 \leq t \leq 1$ (Fig. 7 ). 


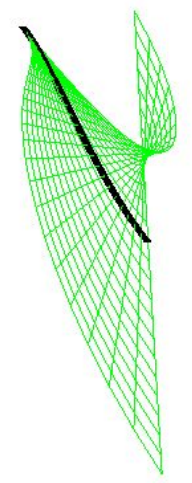

Figure $7 . \Phi_{6}(s, t)$ as a member of the developable ruled surface pencil and its geodesic curve.

Authors Contribution Statement The authors contributed equally. All authors read and approved the final copy of the manuscript.

Declarartion of Competing Interests The authors declare that they have no known competing financial interests or personal relationships that could have appeared to influence the work reported in this paper.

Acknowledgement The authors would like to thank the referees for their helpful suggestions and their valuable comments which helped to improve the manuscript.

\section{REFERENCES}

[1] Bechmann, D., Gerber, D., Arbitrary shaped deformation with dogme, Visual Comput., 19 ( 2-3) (2003), 175-186.

[2] Peng, Q., Jin, X., Feng, J., Arc-length-based axial deformation and length preserving deformation, In Proceedings of Computer Animation., (1997), 86-92.

[3] Lazarus, F., Coquillart, S., Jancène, P., Interactive axial deformations, In Modeling in Computer Graphics., Springer, Verlag, 1993.

[4] Lazarus, F., Verroust, A., Feature-based shape transformation for polyhedral object, In Proceedings of the 5th Eurographics Workshop on Animation and Simulation., (1994), 1-14.

[5] Lazarus, F., Coquillart, S., Jancène, P., Axial deformation: an intuitive technique, Comput. Aid. Des., 26 (8) (1994), 607-613.

[6] Llamas, I., Powell, A., Rossignac, J., Shaw, C.D., Bender : A virtual ribbon for deforming $3 \mathrm{~d}$ shapes in biomedical and styling applications, In Proceedings of Symposium on Solid and Physical Modeling., (2005), 89-99. 
[7] Bloomenthal,M., Riesenfeld, R.F., Approximation of sweep surfaces by tensor product NURBS, In SPIE Proceedings Curves and Surfaces in Computer Vision and Graphics., 2 (1610) (1991), 132-154.

[8] Pottmann, H., Wagner, M., Contributions to motion based surface design, Int. J. Shape Model., 4 (3\&4 ) (1998), 183-196.

[9] Siltanen, P., Woodward, C., Normal orientation methods for 3D offset curves, sweep surfaces, skinning, In Proceedings of Eurographics., (1992), 449-457.

[10] Wang,W., Joe, B., Robust computation of rotation minimizing frame for sweep surface modeling, Comput. Aid. Des., 29 (1997), 379-391.

[11] Shani, U., Ballard, D.H., Splines as embeddings for generalized cylinders. Comput. Vision Graph. Image Proces., 27 (1984), 129-156.

[12] Bloomenthal, J., Modeling the mighty maple, In Proceedings of SIGGRAPH., (1985), 305311.

[13] Bronsvoort, W.F., Klok, F., Ray tracing generalized cylinders, ACM Trans. Graph.,4 (4) (1985), 291-302.

[14] Semwal, S.K., Hallauer, J., Biomedical modeling: implementing line-of-action algorithm for human muscles and bones using generalized cylinders, Comput. Graph., 18 (1) (1994), 105112.

[15] Banks, D.C., Singer, B.A., A predictor-corrector technique for visualizing unsteady flows, IEEE Trans on Visualiz. Comput. Graph., 1 (2) (1995), 151-163.

[16] Hanson, A.J., Ma, H., A quaternion approach to streamline visualization, IEEE Trans Visualiz. Comput. Graph., 1 (2) (1995), 164-174.

[17] Hanson, A., Constrained optimal framing of curves and surfaces using quaternion gauss map. In Proceedings of Visulization., (1998), 375-382.

[18] Barzel, R., Faking dynamics of ropes and springs, IEEE Comput. Graph. Appl., 17 (3) (1997), 31-39.

[19] Jüttler, B., Rational approximation of rotation minimizing frames using Pythagoreanhodograph cubics, J. Geom. Graph., 3 (1999), 141-159.

[20] Bishop, R. L., There is more than one way to frame a curve, Ame. Math. Mon., 82 (1975), 246-251.

[21] O'Neill, B., Elementary Differential Geometry, Academic Press Inc., New York, 1966.

[22] Farouki, R.T., Sakkalis, T., Rational rotation-minimizing frames on polynomial space curves of arbitrary degree, J. Symbolic Comput., 45 (2010), 844-856 .

[23] Brond, R., Jeulin, D., Gateau, P., Jarrin, J., Serpe, G., Estimation of the transport properties of polymer composites by geodesic propagation, J. Microsc., 176 (1994), 167-177.

[24] Bryson, S.,Virtual spacetime: an environment for the visualization of curved spacetimesvia geodesic flows, Technical Report, NASA NAS., Number RNR-92 (1992).

[25] Grundig, L., Ekert, L., Moncrieff, E., Geodesic and semi-geodesic line algorithms for cutting pattern generation of architectural textile structures, In: Lan TT, editor. Proceedings of the Asia-Pacific Conference on Shell and Spatial Structures, Beijing, 1996.

[26] Haw, R.J., An application of geodesic curves to sail design, Comput. Graphics Forum., 4(2) (1985), 137-139.

[27] Haw, R.J., Munchmeyer, R.C., Geodesic curves on patched polynomial surfaces, Comput. Graphics Forum., 2 (4) (1983), 225-232.

[28] Wang, G.J., Tang, K., Tai, C.L., Parametric representation of a surface pencil with a common spatial geodesic. Comput. Aided Des., 36 (5) (2004), 447-459.

[29] Deng, B., Special Curve Patterns for Freeform Architecture Ph.D. thesis, Eingereicht an der Technischen Universitat Wien, Fakultat für Mathematik und Geoinformation von , 2011.

[30] Kasap, E., Akyıldız, F.T., Orbay, K., A generalization of surfaces family with common spatial geodesic, Appl. Math. Comput., 201 (2008), 781-789. 
[31] Kasap, E., Akyildiz, F.T., Surfaces with common geodesic in Minkowski 3-space, Appl. Math. Comput., 177 (2006), 260-270.

[32] Şaffak, G., Kasap, E., Family of surface with a common null geodesic, International Journal of Physical Sciences., 4 (8) (2009), 428-433.

[33] Atalay, G.S., Kasap, E., Surfaces family with common null asymptotic, Appl. Math. Comput., doi: 10.1016/J.amc.2015.03.067.

[34] Bayram, E., Güler, F., Kasap, E., Parametric representation of a surface pencil with a common asymptotic curve, Comput. Aided Des., 44 (2012), 637-643.

[35] Bayram, E., Bilici, M., Surfaces family with a common involute asymptotic curve, International Journal of Geometric Methods in Modern Physics., 13(5) (2016).

[36] Atalay, G.S., Surfaces family with a common Mannheim geodesic curve, Journal of Applied Mathematics and Computation., 2 (4) (2018), 155-165.

[37] Atalay, G.S., Surfaces family with a common Mannheim asymptotic curve, Journal of Applied Mathematics and Computation., 2 (4) (2018), 143-154.

[38] Ayvacı, K.H., Ortak Mannheim-B İsogeodezikli ve İsoasimptotikli Yüzey Ailesi, Ondokuz Mayıs Üniversitesi Fen Bilimleri Enstitüsü, Yüksek Lisans Tezi (Basılmış), 2019.

[39] Ayvacı, K.H., Atalay, G.S., Surface Family With A Common Bertrand-B Isogeodesic Curve, Journal of the Institute of Science and Technology., 10 (3) (2020), 1975-1983.

[40] Do Carmo, M.P., Differential geometry of curves and surfaces, Prentice Hall, Inc., Englewood Cliffs, New Jersey, 1976.

[41] Klok, F., Two moving coordinate frames along a 3D trajectory, Comput. Aided Geom. Design., 3 (1986), 217-229.

[42] Han, C.Y., Nonexistence of rational rotation-minimizing frames on cubic curves, Comput. Aided Geom. Design., 25 (2008), 298-304.

[43] Li ,C.Y., Wang, R.H., Zhu, C.G., An approach for designing a developable surface through a given line of curvature, Comput. Aided Des., 45 (2013), 621-627. 